Proceedings

\title{
A Novel Strategy to Achieve Enhanced Reinforcement and Decreased Damping in CNT-Nanocomposites ${ }^{+}$
}

\author{
Maryam Karimzadeh 1,2, Michela Talò ${ }^{2}$, Walter Lacarbonara ${ }^{2}$ and Giulia Lanzara 1,* \\ 1 Engineering Department, University of Rome, Roma Tre, 00146 Rome, Italy; karimzade_m@yahoo.com \\ 2 Structural and Geotechnical Engineering Department, Sapienza University of Rome, 00184 Rome, Italy; \\ michela.talo@uniroma1.it (M.T.); walter.lacarbonara@uniroma1.it (W.L.) \\ * Correspondence: giulia.lanzara@uniroma3.it \\ + Presented at the 18th International Conference on Experimental Mechanics (ICEM18), Brussels, Belgium, \\ 1-5 July 2018.
}

Published: 5 June 2018

\begin{abstract}
In recent decades, polymer-carbon nanotube (CNTs) composite materials have drawn much attention for their potential applications as nanofiller in unique lightweight materials with superior mechanical properties. For structural engineering applications, high strength and stiffness can be delivered and conveniently tuned in nanocomposites by ensuring and effective load-transfer at the $\mathrm{CNT} /$ polymer interfaces. To achieve such an improvement, conventional approaches are based on complex chemical functionalization processes. In this work, the mechanical properties of nickel-coated carbon nanotube (Ni-CNT) reinforced polyamic acid-nanocomposites (Ni-CNTs /PAA) are presented and compared to those of pristine CNT nanocomposites (CNTs/PAA) in terms of stiffness, strength and damping capacity. The Ni nanoparticles decorating the CNTs outer walls induce an interlocking mechanism at the $\mathrm{CNTs} /$ matrix interface. A significant increase in the elastic modulus is thus observed for this kind of materials. On the other hand, the Ni-CNTs/PAA nanocomposite, decreases its damping capacity when compared with pristine CNTs/PAA nanocomposite. It is worth noting that the two investigated nanocomposites were conceived with the same total weight fraction of nanofiller, by assuming as nanofiller the pristine CNTs and the $\mathrm{Ni}$-coated CNTs, respectively. The recorded stiffness enhancement and damping reduction are reached despite the significantly smaller amount of CNTs contained in the Ni-coated CNT nanocomposite samples. Indeed, for these nanocomposites, Ni nanoparticles, with their higher mass density, represent the $60 \mathrm{wt} \%$ of the total nanofiller weight. The results proved the concept that when metal nanoparticles coat the CNTs outer walls, better CNTs/matrix adhesion can be achieved without the need to undergo complex CNTs functionalization procedures. This is an advantage since chemical functionalization typically shows the drawback of damaging the CNTs, by introducing defects on their outer walls.
\end{abstract}

Keywords: carbon nanotube; nanocomposites; metal nanoparticles CNT coating; interlocking; strength

\section{Introduction}

The unique mechanical, electrical, and optical properties [1-4] of multiwall carbon nanotubes (MWNTs) made them very attractive for the fabrication of new advanced materials, particularly nanofiller/polymer composites with improved and tailored properties. Several investigations on the mechanical properties of the MWNT nanocomposites showed that an effective beneficial utilization of carbon nanotubes (CNTs) in composite applications strongly depends on the ability to disperse 
homogeneously the CNTs throughout the matrix [5-7]. Good interfacial bonding and/or interaction forces between nanotubes and polymers are the necessary conditions for improving the mechanical response of composites. A significant number of different surface treatments and functionalization techniques have been devised for improving the dispersion and interfacial adhesion between the CNTs and polymer matrices. As a result, the treated composites were proved to exhibit higher stiffness and strength than those without treatment due to the formation of stronger interfacial bonds and more homogeneous dispersion of CNTs [8].

In this work, a magnetic metal coating of the carbon nanotubes is employed as a novel interface engineering approach to improve the CNT/matrix adhesion. In particular, carbon nanotubes decorated with $10 \mathrm{~nm}$-diameter nickel nanoparticles are dispersed in a polyamic acid (PAA) hosting matrix.

Recently, researchers have attempted to deposit metals or metal compounds onto the CNTs surface aiming at improving the magnetic properties of nanocomposites. This kind of nanocomposites have potential applications in various engineering fields, such as magnetic recording, magnetic data storage devices, toners and inks for xerography, and magnetic resonance imaging. Therefore, studies on magnetic nanocomposites, especially on magnetic CNTs composites, are rapidly expanding.

Despite several studies on the metal-coated CNT nanocomposites [9-12] were carried out, there is a lack of the mechanical response investigations for Ni-coated CNTs in polymer matrices [13]. In this study, we investigate the mechanical response of polyamic acid (PAA) nanocomposites that are reinforced with nickel coated MWNTs. Pristine CNT/PAA and Ni-CNT/PAA nanocomposites are prepared by solvent casting technique and their stiffness, strength and damping capacity are experimentally estimated.

\section{Fabrication Process}

The following materials were used: MWNTs and Nickel-coated MWCNTs (60 wt\% Nickel; 38 wt $\%$ MWCNTs) with an outer diameter of 50-80 nm; N-Methyl-2-pyrrolidinone (NMP) solvent; and polyamic acid (PAA).

A $12 \mathrm{wt} \%$ of nanofiller (MWCNTs and Ni-MWCNTs, respectively) was dispersed in the NMP solvent and the resulting suspension sonicated for $45 \mathrm{~min}$, while polyamic acid and NMP were mixed through magnetic stirring. The PAA solution was added to the MWCNT/NMP suspension and sonicated for $20 \mathrm{~min}$. The obtained MWCNT/PAA/NMP dispersion was cast onto glass slides and dried in vacuum oven at $95^{\circ} \mathrm{C}$ for $1.5 \mathrm{~h}$ to remove the solvent.

\section{Mechanical Characterization}

Thin nanocomposite rectangular specimens were obtained by cutting the nanocomposite films in stripes of $3.5 \mathrm{~mm}$ in width and $15 \mathrm{~mm}$ in length, while their thickness was measured to be between 35 and $50 \mu \mathrm{m}$. A dynamic mechanical analyzer DMAQ800 was employed to perform monotonic and cyclic tensile tests by using the tension film clamp. A preload of $0.1 \mathrm{~N}$ was first applied, then a force rate of $2 \mathrm{~N} / \mathrm{min}$ was set for both the monotonic and cyclic tensile tests. In the monotonic tensile tests, useful to characterize the materials elastic regions, a maximum strain of $5 \%$ was reached. The loading/unloading tensile tests were performed in the elastic region and, two cycles at the maximum stress amplitudes of $15 \mathrm{MPa}$ and $20 \mathrm{MPa}$, respectively, were obtained. The tests were repeated on 5 specimens for each nanocomposite material.

\section{Results and Discussion}

The representative stress-strain curves are reported in Figure 1. As expected, the nanocomposite with nickel-coated CNTs shows a significantly enhanced mechanical response with respect to the pristine CNT/PAA nanocomposite. When the two nanocomposite materials are loaded, they exhibit an elastic behavior up to a strain of 1\%. A Young modulus of $1.497 \mathrm{GPa}$ and $1.936 \mathrm{GPa}$ are observed for the pristine CNT/PAA and Ni-CNT/PAA nanocomposites, respectively. 
Such outcome proves the effectiveness of the nickel nanoparticles which increase the density of the van der Waals interaction forces at the CNT/matrix interfaces. A $29 \%$ improvement of the elastic modulus is delivered in the interface-engineered nanocomposite with respect to the nanocomposite with unmodified interfaces.

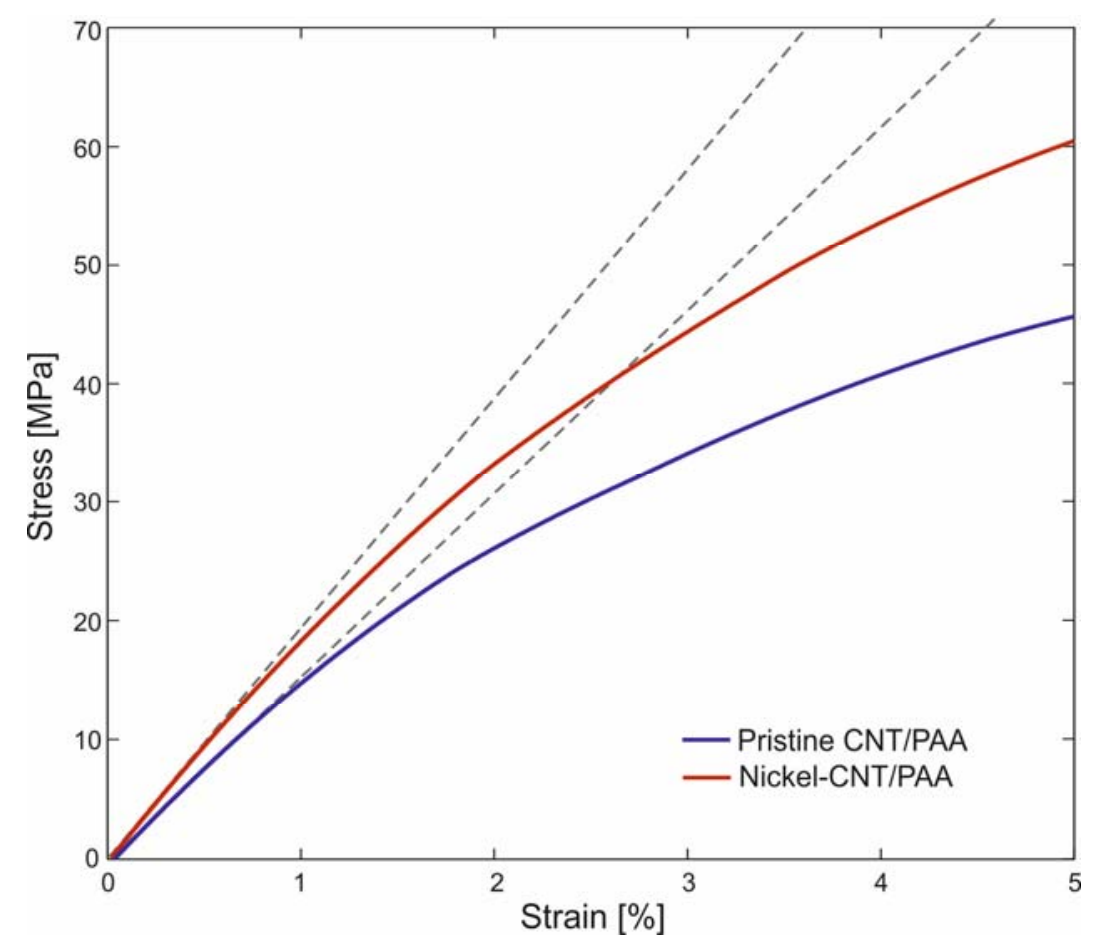

Figure 1. Monotonic tensile tests at a constant force rate of $2 \mathrm{~N} / \mathrm{min}$ for the pristine CNT/PAA nanocomposite and nickel-coated CNT/PAA nanocomposite. The representative stress-strain curves are obtained by averaging and interpolating the test data of five specimens per each material.

The loading/unloading stress-strain curves are reported in Figure 2, performed in the materials elastic region (below 1.5\% strain). Besides the reinforcing effect provided by the nickel coating of the CNTs, an additional effect on the nanocomposite damping capacity is also observed. The nickel-CNT/PAA nanocomposites show narrower hysteretic cycles with respect to the pristine CNT/PAA nanocomposites. The hysteresis, arising in nanocomposites with nickel nanoparticles interface modification, is mainly due to the viscoelastic behavior of the PAA matrix characterized by free and short oligomer chains. Indeed, PAA chains show high mobility and are kept together by weak interaction forces. The PAA chains easily slide each other and adjust their configuration once loads are applied. On the other hand, the PAA chains near to the nickel-coated CNTs must interact with the nickel nanoparticles and transfer the load to the CNT nanofiller through them. Due to the stronger electrostatic interaction forces between the polar PAA oligomer chains and the metallic nanoparticles, the nickel-CNT/PAA nanocomposite is characterized by strong interfaces with good adhesion between matrix and nanofiller.

The hysteresis in the pristine CNT/PAA nanocomposites is instead the result of two contributions, i.e., (i) the contribution of the PAA matrix inter-chains sliding, and (ii) the contribution of the interfacial frictional sliding between the CNTs and the PAA chains, a phenomenon well known as stick-slip [14]. This energy dissipation phenomenon observed in pristine CNT/PAA nanocomposites is easily activated due to the weak adhesion at the unmodified CNT/matrix interfaces. Low interfacial shear stress levels are required to overcome the weak interfacial interaction forces and trigger the slippage of the PAA chains with respect to the CNTs. 


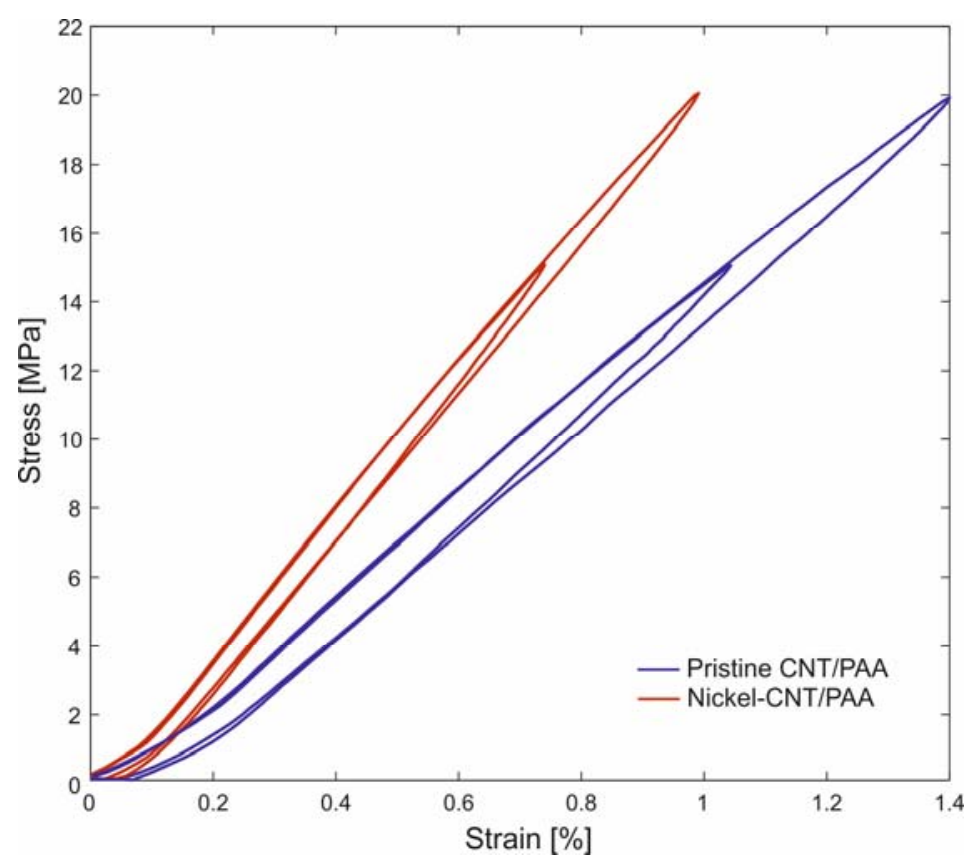

Figure 2. Cyclic tensile tests at a constant force rate of $2 \mathrm{~N} / \mathrm{min}$ for the pristine CNT/PAA nanocomposite and nickel-coated CNT/PAA nanocomposite. Loading/unloading cycles at two stress amplitudes (15 $\mathrm{MPa}$ and $20 \mathrm{MPa}$, respectively) are obtained per each material. .

In particular, the equivalent damping ratio of the two nanocomposite materials is computed by directly measuring the dissipated energy as the area enclosed by the loading/unloading cycle, and the elastic strain energy as the area under the loading branch. The equivalent damping ratios are found to be $1.418 \%$ and $1.064 \%$ for the pristine CNT/PAA and nickel-CNT/PAA nanocomposites, respectively. Therefore, the interface-engineered nanocomposites have a reduced damping capacity of $33 \%$ compared to the pristine nanocomposites. Such a response is due to the strengthening effect of the nickel nanoparticles which increase the interfacial shear stresses needed to activate the stick-slip energy dissipation phenomenon.

The carried out mechanical tests proved the effectiveness of the selected interface engineering approach. By exploiting nickel nanoparticles, a significant enhancement in the nanocomposite elastic response is observed. The nickel nanoparticles CNTs coating has the advantage of not damaging the CNTs outer walls structure, in contrast to other interface treatments, such as covalent functionalization. Covalent functionalization in fact requires the use of acids to create defects sites on the CNT surface and attach functional groups [15]. Furthermore, it is worth to point out that the CNT weight fraction in the nickel-CNT/PAA nanocomposites is only the $40 \%$ of the weight fraction of CNTs employed in the pristine CNT/PAA nanocomposites. Indeed, the interface modified nanocomposites were fabricated by replacing the weight of pristine filler with the same weight of modified filler. Therefore, the weight of the modified filler is in part given by the heavy nickel nanoparticles (i.e., $60 \%$ of the weight is due to nickel). This strategy was employed in order to compare two ultra-lightweight nanocomposite materials with the same total weight. Although $\mathrm{Ni}-\mathrm{CNT} / \mathrm{PAA}$ nanocomposite contain less carbon nanotubes, thus, there is less interfacial surface area available for the load transfer mechanism, excellent enhancements in the material mechanical properties are obtained.

\section{Conclusions}

In this paper, it is shown that metal (Ni) nanoparticles can successfully be used as an alternative method to improve the effectiveness of the load transfer mechanism between the CNTs and a hosting matrix. In this case study, the matrix is constituted of short oligomer chains, that, together with the $\mathrm{Ni}$ nanoparticles, create an interlocking mechanism enhancing the nanocomposite mechanical response. The improvement in CNT-matrix interfacial properties provides a stiffness 
increase and a damping capacity decrease. In particular, Ni-CNTs nanocomposites show a $29 \%$ improvement in elastic modulus and a 33\% decrease in damping. The advantage of this approach is that the CNTs can express their reinforcement effect without being subject to harsh treatments that damage their structure, as in chemical functionalization.

Author Contributions: G.L. conceived and designed the experiments; M.K. fabricated the nanocomposite samples; M.T. performed the experiments and analyzed the data; G.L., M.T. and W.L. interpreted the data; M.K., M.T. and G.L. wrote the paper.

Acknowledgments: This work was partially supported by the European Office of Aerospace Research and Development/Air Force Office of Scientific Research Grant (Grant No. FA9550-14-1-0082 DEF), and by the European Research Council under the European Union's Seventh Framework Program (FP/2007-2013)/ERC Grant Agreement No. 308261. David Garner, EOARD program manager for this Grant, and "Les" Lee, AFOSR "Multifunctional materials and microsystems" program manager, are gratefully acknowledged for their support.

\section{References}

1. Yu, M.-F.; Lourie, O.; Dyer, M.J.; Moloni, K.; Kelly, T.F.; Ruoff, R.S. Strength and breaking mechanism of multiwalled carbon nanotubes under tensile load. Science 2000, 287, 637-640.

2. Ajayan, P.M. Nanotubes from carbon. Chem. Rev. 1999, 99, 1787-1799.

3. Rao, C.N.R.; Govindaraj, B.A.; Nath, M. Nanotubes. ChemPhysChem 2001, 2, 78-105.

4. Demczyk, B.G.; Wang, Y.M.; Satishkumar, J.C.; Cumings, M.H.; Han, W.; Zettl, A.; Ritchie, R.O. Direct mechanical measurement of the tensile strength and elastic modulus of multiwalled carbon nanotubes. Mater. Sci. Eng. A 2002, 334, 173-178.

5. Ruan, S.L.; Gao, P.; Yang, X.G.; Yu, T.X. Toughening high performance ultrahigh molecular weight polyethylene using multiwalled carbon nanotubes. Polymer 2003, 44, 5643-5654.

6. Andrews, R.; Jacques, D.; Qian, D.; Rantell, T. Multiwall carbon nanotubes: synthesis and application. Acc. Chem. Res. 2002, 35, 1008-1017.

7. Rouse, J.H.; Lillehei, P.T. Electrostatic assembly of polymer/single walled carbon nanotube multilayer films. Nano Lett. 2003, 3, 59-62.

8. Sham, M.L.; Kim, J.K. Surface functionalities of multi-wall carbon nanotubes after UV/Ozone and TETA treatments. Carbon 2006, 44, 768-77.

9. Liang, J.; Li, H.; Qi, L.; Tian, W.; Li, X.; Zhou, J.; Wang, D.; Wei. Influence of Ni-CNTs additions on the microstructure and mechanical properties of extruded Mg-9Al alloy J. Mater. Sci. Eng. A 2016, 678, 101-109.

10. Duan, K.; Li, L.; Hu, Y.; Wang, X. Damping characteristic of Ni-coated carbon nanotube/copper composite. Mater. Des. 2017, 133, 455-463.

11. Billah, M.M.; Chen, Q. J. Sn/MWCNT nanocomposites fabricated by ultrasonic dispersion of Ni-coated MWCNTs in Molten Tin. Electron. Mater. 2018, 47, 2366-2373.

12. Olek, M.; Kempa, K.; Jurga, S.; Giersig, M. Nanomechanical properties of silica-coated multiwall carbon nanotubes/poly(methyl methacrylate)composites. Langmuir 2005, 21, 3146-3152.

13. Zhao, D.; Li, X.; Shen, Z. Microwave absorbing property and complex permittivity and permeability of epoxy composites containing Ni-coated and Ag filled carbon nanotubes. Compos. Sci. Technol. 2008, 68, 2902-2908.

14. Sun, L.; Gibson, R.F.; Gordaninejad, F.; Suhr, J. Energy absorption capability of nanocomposites: A review. Compos. Sci. Technol. 2009, 69, 2392-2409.

15. Ma, P.-C.; Siddiqui, N.A.; Marom, G.; Kim, J.-K. Dispersion and functionalization of carbon nanotubes for polymer-based nanocomposites: A review. Compos. Part A Appl. Sci. Manuf. 2010, 41, 1345-1367.

(C) 2018 by the authors. Licensee MDPI, Basel, Switzerland. This article is an open access article distributed under the terms and conditions of the Creative Commons Attribution (CC BY) license (http://creativecommons.org/licenses/by/4.0/). 